\title{
SÍFILIS GESTACIONAL Y FACTORES ASOCIADOS EN HOSPITALES PÚBLICOS DEL PERÚ EN EL PERIODO 2000-2010
}

\author{
Gustavo F. Gonzales ${ }^{1,2, a}$, Vilma Tapia ${ }^{2, b}$, Suzanne J. Serruya ${ }^{3, c}$
}

\begin{abstract}
RESUMEN
Objetivos. Determinar el perfil epidemiológico de la sífilis gestacional y factores asociados en una población peruana. Materiales y métodos. El estudio es un análisis secundario de datos obtenidos de hospitales públicos del Perú para el periodo 2000-2010. Se incluyó datos de 652636 gestantes de 37 ciudades y de 45 establecimientos de salud del Perú. Resultados. Se estimó la prevalencia de sífilis gestacional en $0,7 \%$ y de tamizaje de sífilis materna en $80,7 \%$. La mayor prevalencia de sífilis materna se encontró en la selva baja seguida de la selva alta. En las tres regiones del Perú se aprecia una disminución de la prevalencia de sífilis gestacional en el transcurso de los años. Las prevalencias al 2010 son de $0,2 \%$ en la costa, $0,23 \%$ en la sierra y $0,47 \%$ en la selva. La ausencia de educación, el bajo número de control prenatal, la mayor paridad, el ser conviviente o soltera, y el vivir en la selva, son factores asociados a la positividad en la prueba de sífilis. Conclusiones. Los resultados sugieren que la prevalencia de sífilis gestacional ha presentado una tendencia decreciente durante la década 2000-2010.
\end{abstract}

Palabras clave: Sifilis; Embarazo; Epidemiología; Syphilinum; Perú, ubicaciones geográficas (fuente: DeCS BIREME).

\section{GESTATIONAL SYPHILIS AND ASSOCIATED FACTORS IN PUBLIC HOSPITALS OF PERU DURING 2000-2010}

\begin{abstract}
Objectives. To determine the epidemiological profile of gestational syphilis and associated factors in a Peruvian population. Materials and methods. The study is a secondary analysis of data obtained from public hospitals in Peru for the 2000-2010 period. 652,636 pregnant women were included from 37 cities and 45 health centers in Peru. Results. The prevalence of gestational syphilis was $0.7 \%$, while $80.7 \%$ reported screening for gestational syphilis. The highest prevalence of maternal syphilis is found in the lowland rainforest followed by the highland rainforest. In the three regions of Peru decreased prevalence of gestational syphilis has been seen throughout the years. The prevalence for 2010 are $0.2 \%$ on the coast, in the highlands $0.23 \%$ and $0.47 \%$ in the jungle. The lack of education and prenatal care, high parity, cohabiting or single, and living in the jungle, are associated with having a positive syphilis test. Conclusions. The results suggests that prevalence of gestational syphilis has declined over the decade 2000-2010.
\end{abstract}

Key words: Syphilis; Pregnancy; Epidemiology; Syphilinum; Peru, geographic locations (source: MeSH NLM).

\footnotetext{
Departamento de Ciencias Biológicas y Fisiológicas, Facultad de Ciencias y Filosofía, Universidad Peruana Cayetano Heredia. Lima, Perú. Instituto de Investigaciones de la Altura, Universidad Peruana Cayetano Heredia. Lima, Perú.

Centro Latinoamericano de Perinatología/Salud de la Mujer y Reproductiva. (CLAP/SMR) OPS/OMS. Montevideo, Uruguay.

a Doctor en Ciencias y doctor en Medicina; ${ }^{\mathrm{b}}$ Magíster en Epidemiología; ${ }^{\mathrm{c}}$ Doctora en Obstetricia y Ginecología Recibido: 27-02-14 Aprobado: 28-05-14
} 


\section{INTRODUCCIÓN}

A nivel mundial, más de dos millones de mujeres embarazadas son diagnosticadas con sífilis anualmente (1). En la región de las Américas, exceptuando Estados Unidos de América y Canadá, se calcula que se producen unos 460 mil casos de sífilis gestacional, y un número anual de sífilis congénita que oscila entre 164 mil y 344 mil (2). La sífilis no tratada en el embarazo conduce a resultados adversos, tales como muerte fetal tardía, prematuridad, y muerte neonatal, en hasta $80 \%$ de los embarazos afectados ${ }^{(3)}$. Sin embargo, estos resultados son prevenibles a través de tamizaje prenatal, que es de bajo costo, y del tratamiento de las mujeres gestantes en caso de serología positiva ${ }^{(4)}$.

En el 2007, la Organización Mundial de la Salud (OMS) lanzó una iniciativa para eliminar la sífilis congénita ${ }^{(5)}$ por lo que resultan importantes todos los esfuerzos que se hacen con dicha finalidad en las diferentes partes del mundo, particularmente en los países en vías de desarrollo como el nuestro. En Uruguay, el Sistema Informático Perinatal (SIP) desarrollado por el Centro Latinoamericano de Perinatología (CLAP), y que es utilizado como una herramienta de vigilancia clínica, permitió detectar el problema de la sífilis gestacional y congénita en dicho país y permitir su vigilancia ${ }^{(6)}$.

La Oficina Panamericana Sanitaria, con datos del 2003, ha estimado la prevalencia de sífilis en mujeres embarazadas de América Latina en 3,1\% (rango: 0,4-6,2). En orden decreciente, la prevalencia estuvo como sigue: El Salvador 6,2; Paraguay 6,0; Bolivia 4,0; Honduras 3,1; Colombia 2,2; Chile 2,2; Cuba 1,8; Brasil 1,6; Perú 0,8 y Panamá 0,4, por cien gestantes evaluadas ${ }^{(7)}$.

La prevalencia de sífilis en gestantes en Perú se ha podido medir mediante los estudios de vigilancia centinela que se han realizado en puérperas desde el año 1996 al 2002. La prevalencia, según estos estudios, ha variado entre 1,0 hasta $1,7 \%$ entre los años 1996 al 2000 (10).

Con la finalidad de lograr un adecuado monitoreo de la sifilis en la gestante, es necesario conocer su epidemiología en las diferentes regiones. La población del Perú se distribuye en tres regiones geográficas en las cuales también se observan diferencias conductuales en relación a la sexualidad (9). En la selva priman las actividades sexuales a más temprana edad, alta tasa de embarazo en adolescentes, mayor número de parejas sexuales por persona y alta tasa global de fecundidad (11). Estos hallazgos se asocian también a prevalencias diferentes de sífilis. En una muestra poblacional de bajos ingresos en ciudades de la costa del Perú se ha estimado una prevalencia de sífilis de $0,5 \%$ en hombres y de $1 \%$ en mujeres ${ }^{(8)}$, mientras que en la Amazonía la prevalencia de sífilis es alta entre hombres $(3,2 \%)$ y mujeres $(2,7 \%)^{(12)}$. Estas cifras contrastan con las observadas en cuatro ciudades de la costa del Perú (Lima, Ica, Trujillo y Chiclayo), donde se ha demostrado una prevalencia de $1,6 \%$ de sífilis en las parejas varones de mujeres embarazadas ${ }^{(13)}$.

Aunque la prevalencia de sifilis gestacional en el Perú está entre las más bajas de la región, es necesario mantener la vigilancia para tratar de erradicarla y, por ello, es importante conocer cómo se desarrolla en las diferentes regiones geográficas del país. Son objetivos del estudio calcular la prevalenciade sífilis en cada región del país; examinar la prevalencia de sífilis en las diferentes zonas del oriente peruano, donde la tasa global de fecundidad es más alta; examinar la prevalencia de sífilis en función de la altitud en que ocurren los embarazos, y evaluar qué factores están asociados a la prevalencia de sífilis gestacional en el Perú.

La OMS está actualmente desarrollando un sistema con el fin de monitorear los esfuerzos para eliminar el impacto global de la sífilis congénita. La proporción de muerte fetal tardía atribuible a sífilis congénita puede ser usado como un indicador para tal monitoreo y evaluación. Sin embargo, este indicador no ha sido nunca validado en el campo. Debido a que el sistema informático perinatal (SIP) contiene datos de las mujeres a través del embarazo y del parto, y es usado en múltiples países de América Latina, es potencialmente una fuente rica en datos para monitorizar la sífilis en el embarazo y efectos asociados en esta región.

\section{MATERIALES Y MÉTODOS}

\section{DISEÑO DEL ESTUDIO}

El diseño es observacional retrospectivo de cohortes. El estudio es un análisis secundario de datos de las bases de datos del SIP de diferentes hospitales públicos del Perú para evaluar la prevalencia de sífilis durante el embarazo y factores asociados. El periodo de las bases de datos corresponden del 1 de enero de 2000 hasta el 2010, obtenidos de distintos hospitales públicos localizados en las tres regiones geográficas del Perú: costa, sierra y selva.

\section{SISTEMA INFORMATICO PERINATAL}

El presente estudio se basa en análisis secundarios de datos del SIP, una herramienta desarrollada en el CLAP en 1983 y utilizada actualmente en once países de América Latina. Este sistema permite tener datos 
desde la primera visita prenatal hasta el parto. En el Perú se ha introducido una versión local del SIP en los hospitales públicos en 1985 y una versión actualizada y generada en un software que es utilizada desde el 2000 (SIP-2000).

La información registrada en este reporte se ha colectado de 45 hospitales de un total de 142 existentes en el Perú, según informes de la página web del Ministerio de Salud para el año 2010. En estos 45 hospitales están representadas la mayoría de regiones administrativas del país y de las tres regiones geográficas. Esto representa una cobertura del $31,7 \%$ de hospitales.

\section{POBLACIÓN DE ESTUDIO}

La población procede de partos consecutivos (madres y recién nacidos) ocurridos en hospitales públicos de diferentes ciudades localizados en costa, sierra y selva del Perú. El estudio incluirá un análisis secundario de datos de las gestantes obtenidos del SIP-2000.

En cada lugar de estudio se ha obtenido del servicio correspondiente la base de datos del SIP-2000. Cada base es almacenada en un disco duro y luego son juntadas generándose una gran base de datos que incluyen un total de 652636 gestantes provenientes de 37 ciudades y de 45 establecimientos de salud del Perú registrados entre 2000 y 2010 . Las bases de datos han sido obtenidas tanto en poblaciones de la costa $(n=278$ 919 gestantes), de la sierra ( $n=227969$ gestantes) y de la selva ( $\mathrm{n}=145748$ gestantes) del Perú.

En la costa se ha colectado información de once establecimientos de salud; en la sierra de veintidós establecimientos; en la selva alta de ocho, y en la selva baja de cuatro. Los periodos de recolección de datos han variado según los hospitales.

En el caso de las poblaciones de la sierra, se han incluido localidades de la sierra norte, sierra centro y sierra sur, con la finalidad de tener representación de las diferentes zonas. En la mayoría de estos hospitales se registran los partos de manera regular (continua) en el SIP-2000, desde sus inicios.

En la sierra norte se han colectado datos de los hospitales de Cajamarca, Chachapoyas, Chota, Cutervo y Huaraz. De la sierra centro de Carhuamayo, Cerro Pasco, Huánuco, Huancayo, Huancavelica, Jauja y Junín, y de la sierra sur de Abancay, Arequipa, Ayacucho, Ayaviri, Cusco, Juliaca, Huancané, Puno y Quillabamba.

En el caso de la selva, se ha clasificado como selva alta y selva baja. Se considera como selva alta cuando la localidad está ubicada entre 400 y 2000 m de altitud, en la zona oriental con respecto a la cordillera de los Andes. Selva baja se define cuando la localidad se encuentra en la zona oriental del país por debajo de los $400 \mathrm{~m}$ de altitud. De la selva alta se han estudiado gestantes de Bagua, La Merced, Oxapampa, Satipo, Tarapoto ${ }^{(2)}$, Tingo María y Villa Rica. De la selva baja se han colectado datos de dos hospitales de lquitos (Loreto), y de Pucallpa y Yarina (Ucayali).

\section{DATOS REGISTRADOS}

Se colectó información acerca de las características sociodemográficas, biológicas y antecedente obstétrico de las madres y datos relacionados a las complicaciones del embarazo actual, y las mediciones de VDRL sérico.

Variable dependiente: la variable principal del estudio, sífilis gestacional, es definida como "Toda mujer embarazada con prueba no treponémica positiva o reactiva. Esta variable es dicotómica (sí, no) y se evalúa como: presencia o ausencia.

El diagnóstico serológico fue realizado mediante las pruebas no treponémicas VDRL (Venereal Disease Research Laboratory) y RPR (Rapid Plasma Reagin). Para confirmar un resultado dudoso de una prueba no treponémica se emplea la técnica FTA-Abs (fluorescent treponemal antibody absorption).

Variables Independientes: las variables que se estudiaron como posibles factores asociados a la presencia de sífilis gestacional fueron a) Variables demográficas: edad materna, nivel de educación, región geográfica (costa, sierra, selva); b) Salud general: peso preembarazo y talla (se calcula índice de masa corporal, IMC); c) Embarazos previos: número de embarazos previos; d) Control prenatal (CPN): número de visitas $<24$ semanas de gestación o $\geq 24$ semanas de gestación: e) Edad gestacional (EG): fecha de embarazo en semanas definida por fecha de última regla (FUR) y por método de Capurro.

La edad materna se define como años completos al tiempo del parto. La educación de la madre se estratificará como ninguna, escuela primaria, secundaria, y estudios superiores. La talla de la madre y el peso antes del embarazo se ha registrado en la primera visita antenatal en centímetros y kilogramos, respectivamente. Con estos datos se ha calculado el IMC como peso preembarazo en kilogramos dividido por talla en metros al cuadrado.

El nivel de educación de la madre se define como el nivel de estudios obtenido al momento del 
embarazo. Es una variable categórica: ninguna (sin estudios), primaria, secundaria, y estudios superiores (universitario, técnico).

Estado civil se define como el estado marital de la gestante.

Región geográfica: definida como áreas con características geográficas y climatológicas diferentes. Es una variable categórica: costa, sierra y selva.

El cuidado prenatal se considera como ninguno, 1-5 visitas, $y>5$ visitas. Los casos previos de bajo peso al nacer y de muerte perinatal son dicotomizados como ausente y presente. La paridad se define como el número de partos previos, incluyendo muerte fetal tardía. El intervalo intergenésico también se registra en el SIP y se define como el número de años entre dos nacimientos. El periodo intergenésico expresado en meses fue calculado cruzando la fecha del último parto/aborto y la FUR.

Edad gestacional del tamizaje de sífilis: definida como la edad del feto al momento de la medición de sífilis. Variable dicotómica: menor de 24 semanas, mayor de 24 semanas.

Historia de bajo peso al nacer: esto es definido como casos en que las madres en el embarazo actual tuvieron nacimientos previos con peso al nacer menores de $2500 \mathrm{~g}$.

\section{MANEJO DE LOS DATOS}

La base de datos contiene información de embarazos en curso (mujeres que en el momento del recojo de la información están gestando), partos y abortos. Para el estudio se ha considerado gestantes con datos de, al menos, una medición de serología para sífilis. Se calcula en cada lugar la prevalencia de tamizaje de sífilis materna (número de mujeres con serología/número de mujeres en la base de datos). La base de datos de cada establecimiento fue evaluada por separado. Al final se integró en una sola base.

La edad gestacional por FUR y por examen clínico (Capurro) presentaron menor proporción de datos faltantes $(<3 \%)$. Los datos faltantes de EG por FUR fueron completados con los datos de EG por examen físico. Al cruzar estas dos variables se observó una alta similitud en estos datos ( \pm 2 semanas). Un número bastante menor $(0,01 \%)$ no tenían registrado la EG ni por FUR ni por Capurro. En este caso se revisaron la FUR, CPN, mediciones antropométricas de $\mathrm{RN}$, datos que podrían ayudar a determinar de forma indirecta la EG.
La variable principal, sífilis gestacional registraba datos completos. Los datos de las otras variables como edad, educación, edad gestacional al tamizaje, estado civil en las gestantes con medición de serología para sífilis, registraban aproximadamente $0,5 \%$ de datos faltantes. Estos fueron analizados por separado y, al no mostrar diferencias con la categoría referencial, fueron imputados a la categoría de mayor frecuencia.

La prevalencia de sífilis gestacional se calcula del número de mujeres embarazadas que presentaron resultado positivo en la prueba treponémica (incluidas pruebas treponémicas rápidas) o no treponémica positiva o reactiva (numerador) dividido entre el número de mujeres embarazadas con prueba de serología realizada (denominador), multiplicado por 100.

\section{ANÁLISIS ESTADÍSTICO}

El análisis estadístico se realizó utilizando el Programa STATA (Stata versión 10.0) para computadora personal (Stata Corporation, 4905 Lakeway Drive, College Station, TX 77845, USA). Los datos cualitativos fueron expresados como frecuencias relativas (porcentajes). La prevalencia de sífilis materna fue calculada por región y año de parto, con respectivos intervalos de confianza al $95 \%$. Las diferencias entre estas con la prueba Chi cuadrada. El análisis de factores de riesgo asociados a la presencia de sífilis gestacional, se realizó mediante la regresión logística, evaluando primero la relación entre cada variable independiente con la variable sífilis gestacional. Aquellas que obtuvieron un $p<0,1$ y aquellas con plausibilidad biológica ingresaron al modelo final. La regresión múltiple fue realizada para predecir la presencia de sífilis en la gestación en función de las variables consideradas predictoras. Se consideró estadística significativa con un valor de $p<0,05$. La evaluación del modelo fue realizada con la prueba de Hosmer y Lemeshow.

\section{CONSIDERACIONES ÉTICAS}

El estudio ha sido aprobado por el Comité Institucional de Ética de la Universidad Peruana Cayetano Heredia. La confidencialidad de los datos se mantuvo a través de códigos sin identificar a personas.

\section{RESULTADOS}

De la población estudiada, 526452 gestantes presentaron evaluación para sífilis $(80,7 \%)$. En total se han registrado 3680 casos de sífilis materna. En la Tabla 1 se observan los datos de las características de 
Tabla 1. Características de la población de estudio según resultados de serología para sífilis obtenidos de los datos del Sistema Informático Perinatal, Perú 2000 - 2010

\begin{tabular}{|c|c|c|c|c|c|c|}
\hline \multirow{2}{*}{ Variable } & \multicolumn{3}{|c|}{ Serología positiva } & \multicolumn{3}{|c|}{ Serología negativa } \\
\hline & $N=3680$ & $(\%)$ & IC $95 \%$ & $N=522772$ & (\%) & IC $95 \%$ \\
\hline \multicolumn{7}{|l|}{ Edad } \\
\hline$\leq 19$ & 678 & $(18,4)$ & $17,2-19,7$ & 108586 & $(20,7)$ & $20,6-20,8$ \\
\hline $20-34$ & 2492 & $(67,7)$ & $66,7-69,2$ & 352544 & $(67,4)$ & $67,3-67,5$ \\
\hline$>34$ & 510 & $(13,8)$ & $12,7-15,0$ & 61642 & $(11,8)$ & $11,7-11,9$ \\
\hline \multicolumn{7}{|l|}{ Educación } \\
\hline Sin educación & 97 & $(2,64)$ & $2,14-3,20$ & 7573 & $(1,45)$ & $1,42-1,48$ \\
\hline Primaria & 1015 & $(27,6)$ & $26,1-29,0$ & 93798 & $(17,9)$ & $17,8-18,1$ \\
\hline Secundaria & 2152 & $(59,0)$ & $57,4-60,6$ & 318826 & $(60,9)$ & $60,8-61,1$ \\
\hline Superior & 396 & $(10,7)$ & $9,77-11,8$ & 102575 & $(19,6)$ & $19,5-19,7$ \\
\hline \multicolumn{7}{|l|}{ Estado civil } \\
\hline Casada & 482 & $(13,1)$ & $12,0-14,2$ & 96637 & $(18,5)$ & $18,4-18,6$ \\
\hline Conviviente & 2715 & $(73,8)$ & $72,3-75,2$ & 346969 & $(66,4)$ & $66,2-66,5$ \\
\hline Soltera & 483 & $(13,1)$ & $12,0-19,2$ & 79166 & $(15,1)$ & $15,0-15,2$ \\
\hline \multicolumn{7}{|l|}{ Control prenatal } \\
\hline Ninguno & 730 & $(19,8)$ & $18,5-21,1$ & 98389 & $(18,8)$ & $18,7-18,9$ \\
\hline $1-5$ & 1327 & $(36,1)$ & $34,5-37,6$ & 172820 & $(33,1)$ & $32,9-33,2$ \\
\hline$>5$ & 1623 & $(44,1)$ & $42,5-45,7$ & 251563 & $(48,1)$ & $47,9-48,3$ \\
\hline \multicolumn{7}{|l|}{ Paridad } \\
\hline Sin hijos & 1208 & $(32,8)$ & $31,3-34,4$ & 241389 & $(46,2)$ & $46,0-46,3$ \\
\hline $1-3$ & 1979 & $(53,8)$ & $52,1-55,4$ & 244531 & $(46,8)$ & $46,6-46,9$ \\
\hline$\geq 4$ & 493 & $(13,4)$ & $12,3-14,5$ & 36852 & $(7,0)$ & $6,9-7,1$ \\
\hline Bajo peso al nacer previo & 145 & $(3,94)$ & $3,33-4,62$ & 12561 & $(2,4)$ & $2,3-2,4$ \\
\hline Muerte perinatal previo & 309 & $(8,4)$ & $7,52-9,34$ & 15717 & $(3,0)$ & $2,9-3,1$ \\
\hline \multicolumn{7}{|l|}{ Edad gestacional de sífilis } \\
\hline$<24$ semanas & 1957 & $(53,2)$ & $51,6-54,8$ & 285844 & $(54,7)$ & $54,5-54,8$ \\
\hline$\geq 24$ semanas & 1723 & $(46,8)$ & $45,2-48,4$ & 236928 & $(45,3)$ & $45,2-45,4$ \\
\hline
\end{tabular}

la población estudiada. Las gestantes con serología positiva tienen mayor edad, menor nivel educativo, son mayormente convivientes, menor control prenatal, mayor paridad y antecedente de niños con bajo peso al nacer o muerte perinatal previa, que las gestantes con serología negativa.

En la Tabla 2 se muestran las características de la población según región geográfica. Comparativamente con la costa, las gestantes de la sierra tienen menor educación, son convivientes, tienen menos número de visitas prenatales, mayor paridad y mayor antecedente de muerte perinatal. Las gestantes de la selva, comparados con las de la costa, son de menor edad, menor educación, conviviente, menor CPN, mayor paridad, antecedente de bajo peso al nacer y muerte perinatal. Comparado entre sierra y selva, las gestantes de la selva tienen de manera diferenciada mayor antecedente de bajo peso al nacer y menor edad. La prevalencia de la muestra global estudiada para sífilis materna en el Perú es de $0,7 \%$ (Tabla 1). Este valor prácticamente se dobla para la selva $(1,3 \%)$ y es menor en la región geográfica de la sierra $(0,5 \%)$ y la costa $(0,5 \%)$. La falta de tamizaje es de $19,3 \%$ a nivel nacional siendo más bajo en la selva (12,3\%) y más alta en la sierra $(27,7 \%)$ y la costa $(20,2 \%)$.

El análisis de regresión entre la cobertura y serología positiva para cada región muestra que no hay asociación entre cobertura de la serología y prevalencia de serología positiva en costa, sierra y selva.

\section{SÍFILIS MATERNA SEGÚN REGIONES Y A TRAVÉS DEL TIEMPO}

La cobertura para tamizaje de sífilis gestacional tuvo un declive entre 2004 y 2005 y luego se recuperó para el 2010 al alcanzar los niveles del 2000 (Figura 1). En la Figura 2 se presentan los datos de la evolución de la sífilis materna entre los años 2000 y 2010 en las diferentes regiones del país. En todas las regiones se aprecia que hay una importante tendencia a disminuir las prevalencias de sífilis materna a través del tiempo.

En la costa hay una disminución de 1,1\% en el 2000 a $0,2 \%$ en el 2010. Se observan caídas importantes 
Tabla 2. Caracteristicas de la población por región geográfica según registro en el Sistema Informático Perinatal, Perú $2000-2010$

\begin{tabular}{|c|c|c|c|c|c|c|c|c|c|}
\hline \multirow{2}{*}{ Variable } & \multicolumn{3}{|c|}{ Costa } & \multicolumn{3}{|c|}{ Sierra } & \multicolumn{3}{|c|}{ Selva } \\
\hline & $N=222533$ & $(\%)$ & IC 95\% & $\mathrm{N}=176122$ & $(\%)$ & IC $95 \%$ & $\mathrm{~N}=127797$ & $(\%)$ & IC $95 \%$ \\
\hline \multicolumn{10}{|l|}{ Edad } \\
\hline$\leq 19$ & 41352 & $(18,6)$ & $18,4-18,7$ & 32551 & $(18,5)$ & $18,3-18,7$ & 35361 & $\left(27,6^{*}\right)$ & $27,4-27,9$ \\
\hline $20-34^{*}$ & 154873 & $(69,6)$ & $69,4-69,8$ & 120504 & $(68,4)$ & $68,2-68,6$ & 79659 & $(62,3)$ & $62,1-62,6$ \\
\hline$>34^{*}$ & 26308 & $(11,8)$ & $11,7-11,9$ & 23067 & $(13,1)$ & $12,9-13,3$ & 12777 & $(10)$ & $9,83-10,2$ \\
\hline \multicolumn{10}{|l|}{ Educación } \\
\hline Sin educación* & 1963 & $(0,88)$ & $0,84-0,92$ & 4282 & $(2,43)$ & $2,35-2,50$ & 1425 & $(1,12)$ & $1,06-1,17$ \\
\hline Primaria* $^{*}$ & 31429 & $(14,1)$ & $13,9-14,3$ & 34769 & $(19,7)$ & $19,5-19,9$ & 28615 & $(22,4)$ & $22,2-22,6$ \\
\hline Secundaria* & 147801 & $(66,4)$ & $66,2-66,6$ & 93956 & $(53,4)$ & $53,1-53,6$ & 7921 & $(62)$ & $61,7-62,3$ \\
\hline Superior* & 41340 & $(18,6)$ & $18,4-18,7$ & 43115 & $(24,5)$ & $24,3-24,7$ & 18516 & $(14,5)$ & $14,3-14,7$ \\
\hline \multicolumn{10}{|l|}{ Estado civil } \\
\hline Casada* & 48394 & $(21,7)$ & $21,5-21,9$ & 33449 & $(19)$ & $18,8-19,2$ & 15276 & $(12)$ & $11,8-12,2$ \\
\hline Conviviente* & 141487 & $(63,6)$ & $63,4-63,8$ & 114007 & $(64,7)$ & $64,5-64,9$ & 94190 & $(73,7)$ & $73,5-73,9$ \\
\hline Soltera* & 32652 & $(14,7)$ & $14,5-14,8$ & 28666 & $(16,3)$ & $16,1-16,4$ & 18331 & $(14,3)$ & $14,1-14,5$ \\
\hline \multicolumn{10}{|l|}{ Control prenatal } \\
\hline Ninguno & 41194 & $(18,5)$ & $18,3-18,7$ & 31537 & $(17,9)$ & $17,7-18,1$ & 26388 & $(20,6)$ & $20,4-20,8$ \\
\hline $1-5^{*}$ & 69692 & $(31,3)$ & $31,1-31,5$ & 61739 & $(35,1)$ & $34,8-35,3$ & 42766 & $(33,5)$ & $33,2-33,7$ \\
\hline$>5^{*}$ & 111647 & $(50,2)$ & $49,9-50,4$ & 82846 & $(47)$ & $46,8-47,3$ & 58693 & $(45,9)$ & $45,6-46,2$ \\
\hline \multicolumn{10}{|l|}{ Paridad } \\
\hline Sin hijos* & 106996 & $(48,1)$ & $47,8-48,3$ & 83278 & $(47,3)$ & $47,1-47,5$ & 52323 & $(40,9)$ & $40,7-41,2$ \\
\hline $1-3^{*}$ & 105552 & $(47,4)$ & $47,2-47,6$ & 78224 & $(44,4)$ & $44,2-44,6$ & 62734 & $(49,1)$ & $48,8-49,4$ \\
\hline$\geq 4^{*}$ & 9985 & $(4,5)$ & $4,40-4,57$ & 14620 & $(8,3)$ & $8,17-8,43$ & 12740 & $(10)$ & $9,8-10,1$ \\
\hline $\begin{array}{l}\text { Antecedente de bajo } \\
\text { peso al nacer }\end{array}$ & 4954 & $(2,23)$ & $2,16-2,29$ & 3956 & $(2,25)$ & $2,17-2,31$ & 3796 & $\left(2,97^{\star}\right)$ & $2,9-3,1$ \\
\hline $\begin{array}{l}\text { Antecedente de } \\
\text { muerte perinatal* }\end{array}$ & 5287 & $(2,38)$ & $2,31-2,43$ & 5894 & $(3,35)$ & $3,26-3,43$ & 4845 & $(3,79)$ & $3,7-3,9$ \\
\hline \multicolumn{10}{|c|}{ Edada gestacional de sífilis } \\
\hline$<24$ semanas* & 124387 & $(55,9)$ & $55,7-56,1$ & 87901 & $(49,9)$ & $49,7-50,2$ & 75513 & $(59,1)$ & $58,8-59,4$ \\
\hline$\geq 24$ semanas $^{*}$ & 98146 & $(44,1)$ & $43,9-44,3$ & 88221 & $(50,1)$ & $49,8-50,3$ & 52284 & $(40,9)$ & $40,6-41,1$ \\
\hline
\end{tabular}

${ }^{*}$ Chi2: $p<0.01$,comparando entre ellas. Ant de BPN y edad $<20$ años, $p<0.01$ comparando con región selva, no entre costa frente a sierra

entre 2001-2004 y entre 2009 y 2010. En la sierra, la prevalencia de sífilis materna disminuye de 0,7-0,8\% en los años $2000-2001$ a $0,2 \%$ en el 2010. Se observan caídas importantes en la prevalencia de sífilis materna entre 2003-2006 y entre 2009-2010. Las prevalencias de sífilis en la selva es alta en el $2000(2,4 \%)$ y se reduce a $0,5 \%$ en el 2010.

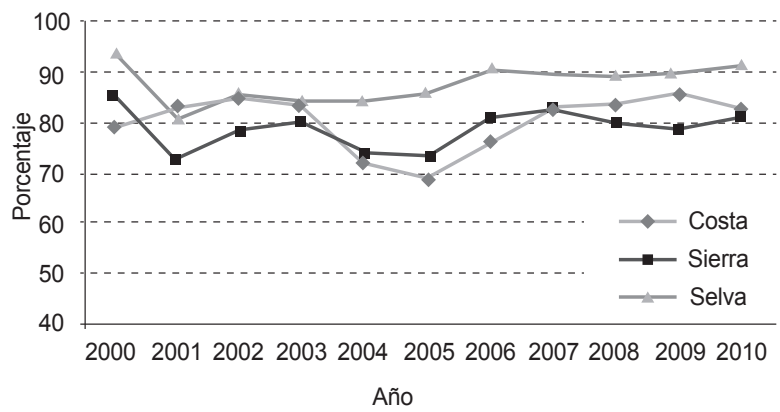

Figura 1. Cobertura de tamizaje (\%) en las tres regiones del Perú entre los años 2000 y 2010
Comparativamente, en el 2000 la prevalencia de sífilis materna era de $1,1 \%$ en la costa, $0,7 \%$ en la sierra, y $2,4 \%$ en la selva. Estas cifras cambian una década después. Así, el 2010, la prevalencia de sífilis materna es de $0,2 \%$ en la costa, $0,2 \%$ en la sierra, y $0,5 \%$ en la selva (Figura 2 ). A nivel nacional hay una importante disminución en la prevalencia de sífilis

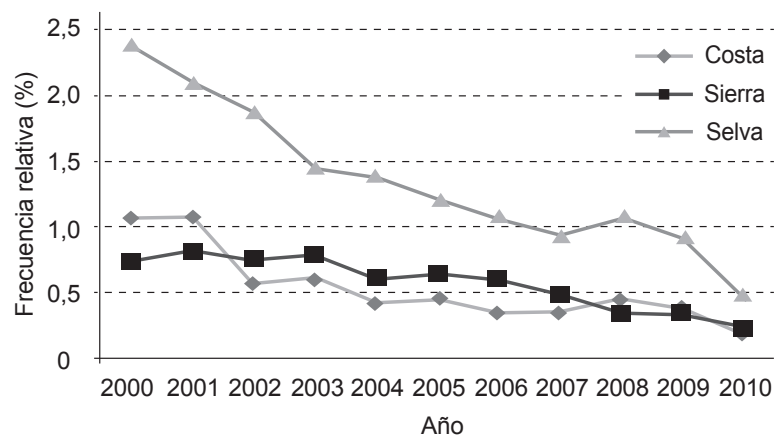

Figura 2. Evolución de la sífilis gestacional en las tres regiones del Perú entre los años 2000 y 2010 


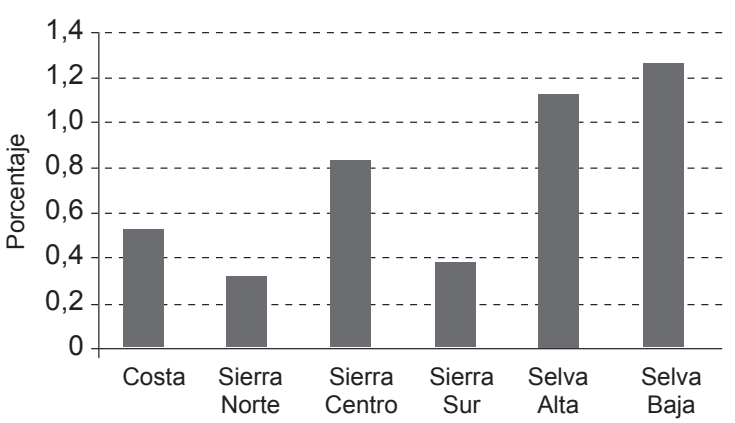

Figura 3. Prevalencia de sífilis materna, según región geográfica, Perú 2000-2010

gestacional al año 2010. La mayor caída se aprecia entre los años 2000 y 2004, y luego la disminución se hace más lenta y gradual. En el año 2010, la cifra de prevalencia de sífilis gestacional en la muestra total del SIP equivale a $0,2 \%$.

\section{PREVALENCIAS DE SIIFILIS MATERNA SEGÚN REGION GEOGRÁFICA Y ESTABLECIMIENTO DE SALUD}

La selva baja es la región geográfica con la más alta prevalencia de sífilis materna $(1,3 \%)$ seguido de la selva alta $(1,1 \%)$. Las prevalencias más bajas se observan en la sierra sur $(0,4 \%)$ y sierra norte $(0,3 \%)$ (Figura 3$)$.

\section{FACTORES ASOCIADOS A SÍFILIS MATERNA SEGÚN REGIÓN GEOGRÁFICA}

En la costa los factores más importantes son tener una muerte perinatal previa $(1,6 \%)$ y la mayor paridad $(1,1 \%)$, en la sierra es la muerte perinatal previa $(1,0 \%)$, ausencia de educación $(0,9 \%)$ y la alta paridad $(0,9 \%)$. En la selva la variable de mayor impacto es la muerte perinatal previa $(3,5 \%)$ y la carencia de nivel educativo $(3,1 \%)$ (Tabla 3).

Tabla 3. Distribución de casos de serología materna positiva para sífilis por región según registro en el Sistema Informático Perinatal, Perú 2000-2010

\begin{tabular}{|c|c|c|c|c|c|c|c|c|c|c|c|}
\hline \multirow{2}{*}{ Variable } & \multicolumn{2}{|c|}{ Población } & \multicolumn{3}{|c|}{ Costa } & \multicolumn{3}{|c|}{ Sierra } & \multicolumn{3}{|c|}{ Selva } \\
\hline & $\mathrm{N}=3680$ & $(\%)$ & $\mathrm{N}=1172$ & $(\%)$ & IC $95 \%$ & $\mathrm{~N}=902$ & $(\%)$ & IC 95\% & $N=1606$ & $(\%)$ & IC 95\% \\
\hline \multicolumn{12}{|l|}{ Edad } \\
\hline$\leq 19$ & 678 & $(0,6)$ & 175 & $(0,4)$ & $0,4-0,5$ & 122 & $(0,4)$ & $0,3-0,4$ & 381 & $(1,1)^{*}$ & $1,0-1,2$ \\
\hline $20-34$ & 2492 & $(0,7)$ & 818 & $(0,5)$ & $0,5-0,6$ & 628 & $(0,5)$ & $0,5-0,6$ & 1046 & $(1,3)^{*}$ & $1,2-1,4$ \\
\hline$>34$ & 510 & $(0,8)$ & 179 & $(0,7)$ & $0,6-0,8$ & 152 & $(0,6)$ & $0,6-0,8$ & 179 & $(1,4)^{*}$ & $1,2-1,6$ \\
\hline \multicolumn{12}{|l|}{ Educación } \\
\hline Sin educación & 97 & $(1,3)$ & 14 & $(0,7)$ & $0,4-1,2$ & 39 & $(0,9)$ & $0,7-1,3$ & 44 & $(3,1)^{*}$ & $2,3-4,1$ \\
\hline Primaria & 1015 & $(1,1)$ & 228 & $(0,7)$ & $0,6-0,8$ & 261 & $(0,8)$ & $0,7-0,9$ & 526 & $(1,8)^{*}$ & $1,7-2,0$ \\
\hline Secundaria & 2152 & $(0,7)$ & 781 & $(0,5)$ & $0,5-0,6$ & 472 & $(0,5)$ & $0,5-0,6$ & 919 & $(1,2)^{*}$ & $1,1-1,2$ \\
\hline Superior & 396 & $(0,4)$ & 149 & $(0,4)$ & $0,3-0,4$ & 130 & $(0,3)$ & $0,3-0,4$ & 117 & $(0,6)^{*}$ & $0,5-0,8$ \\
\hline \multicolumn{12}{|l|}{ Estado civil } \\
\hline Casada & 482 & $(0,5)$ & 210 & $(0,4)$ & $0,4-0,5$ & 148 & $(0,4)$ & $0,4-0,5$ & 124 & $(0,8)^{*}$ & $0,7-1,0$ \\
\hline Conviviente & 2715 & $(0,8)$ & 818 & $(0,6)$ & $0,5-0,6$ & 627 & $(0,6)$ & $0,5-0,6$ & 1270 & $(1,4)^{*}$ & $1,3-1,4$ \\
\hline Soltera & 483 & $(0,6)$ & 144 & $(0,4)$ & $0,4-0,5$ & 127 & $(0,4)$ & $0,4-0,5$ & 212 & $(1,2)^{*}$ & $1,0-1,3$ \\
\hline \multicolumn{12}{|l|}{ Control prenatal } \\
\hline Ninguno & 730 & $(0,7)$ & 216 & $(0,5)$ & $0,5-0,6$ & 209 & $(0,7)$ & $0,6-0,8$ & 305 & $(1,2)^{*}$ & $1,0-1,3$ \\
\hline $1-5$ & 1327 & $(0,8)$ & 406 & $(0,6)$ & $0,5-0,6$ & 333 & $(0,5)$ & $0,5-0,6$ & 588 & $(1,4)^{*}$ & $1,3-1,5$ \\
\hline$>5$ & 1623 & $(0)$, & 550 & $(0,5)$ & $0,6-0,5$ & 360 & $(0,4)$ & $0,4-0,5$ & 713 & $(1,2)^{*}$ & $1,1-1,3$ \\
\hline \multicolumn{12}{|l|}{ Paridad } \\
\hline Sin hijos & 1208 & $(0,5)$ & 445 & $(0,4)$ & $0,4-0,5$ & 282 & $(0,3)$ & $0,3-0,4$ & 481 & $(0,9)^{*}$ & $0,8-1,0$ \\
\hline $1-3$ & 1979 & $(0,8)$ & 620 & $(0,6)$ & $0,5-0,6$ & 493 & $(0,6)$ & $0,6-0,7$ & 866 & $(1,4)^{*}$ & $1,3-1,5$ \\
\hline$\geq 4$ & 493 & $(1,3)$ & 107 & $(1,1)$ & $0,9-1,3$ & 127 & $(0,9)$ & $0,7-1,0$ & 259 & $(2,0)^{*}$ & $1,8-2,3$ \\
\hline Bajo peso al nacer previo & 145 & $(1,1)$ & 40 & $(0,8)$ & $0,4-1,1$ & 36 & $(0,9)$ & $0,6-1,3$ & 69 & $(1,8)^{*}$ & $1,4-2,3$ \\
\hline Muerte perinatal previo & 309 & $(1,9)$ & 84 & $(1,6)$ & $1,3-2,0$ & 58 & $(1,0)$ & $0,7-1,3$ & 167 & $(3,5)^{*}$ & $3,0-4,0$ \\
\hline \multicolumn{12}{|c|}{ Edad gestacional de sífilis } \\
\hline$<24$ semanas & 1957 & $(0,7)$ & 619 & $(0,5)$ & $0,5-0,5$ & 401 & $(0,5)$ & $0,4-0,5$ & 937 & $(1,2)^{*}$ & $1,2-1,3$ \\
\hline$\geq 24$ semanas & 1723 & $(0,7)$ & 553 & $(0,6)$ & $0,5-0,6$ & 501 & $(0,6)$ & $0,5-0,6$ & 669 & $(1,3)^{*}$ & $1,2-1,4$ \\
\hline
\end{tabular}

* Chi2: $p<0,01$ comparando con región selva, entre costa frente a sierra no hay diferencias. 
Tabla 4. Análisis multivariable para determinar factores asociados con la positividad de la serología para sífilis

\begin{tabular}{|c|c|c|}
\hline Variable & $\begin{array}{c}\text { OR } \\
\text { ajustado }\end{array}$ & $p$ \\
\hline \multicolumn{3}{|l|}{ Edad } \\
\hline $20-34$ & 1,00 & \\
\hline$<20$ & $0,97(0,88-1,07)$ & 0,63 \\
\hline$>34$ & $0,9(0,81-1,01)$ & 0,07 \\
\hline \multicolumn{3}{|l|}{ Estudios } \\
\hline Educación secundaria y superior & 1,00 & \\
\hline $\begin{array}{l}\text { No educción y educación } \\
\text { primaria }\end{array}$ & $1,47(1,36-1,58)$ & 0,001 \\
\hline \multicolumn{3}{|l|}{ Control prenatal } \\
\hline$\geq 6$ & 1,00 & \\
\hline Ninguno $0<6$ & $1,09(1,03-1,17)$ & 0,007 \\
\hline \multicolumn{3}{|l|}{ Paridad } \\
\hline $1-3$ & 1,00 & \\
\hline Ninguno & $0,67(0,61-0,73)$ & 0,001 \\
\hline$>3$ & $1,32(1,18-1,48)$ & 0,001 \\
\hline $\begin{array}{l}\text { Antecedente de bajo peso al } \\
\text { nacer }\end{array}$ & $1,27(1,07-1,51)$ & 0,005 \\
\hline $\begin{array}{l}\text { Antecedente de muerte } \\
\text { perinatal }\end{array}$ & $2,13(1,88-2,41)$ & 0,001 \\
\hline \multicolumn{3}{|l|}{ Estado civil } \\
\hline Casada & 1,00 & \\
\hline Conviviente & $1,56(1,42-1,73)$ & 0,001 \\
\hline Soltera & $1,59(1,40-1,83)$ & 0,001 \\
\hline \multicolumn{3}{|l|}{ Región } \\
\hline Costa & 1,00 & \\
\hline Sierra & $0,89(0,82-0,98)$ & 0,018 \\
\hline Selva & $2,06(1,91-2,23)$ & 0,001 \\
\hline \multicolumn{3}{|l|}{ Edad gestacional de sífilis } \\
\hline$<24$ semanas & 1,00 & \\
\hline$\geq 24$ semanas & $1,02(0,96-1,10)$ & 0,48 \\
\hline
\end{tabular}

En la Tabla 3 se muestran la prevalencia de serología positiva por región geográfica y según característica estudiada. No se observan diferencias en las prevalencias de serología positiva de sífilis entre costa y sierra para cada variable evaluada; sin embargo, en la selva, las prevalencias de sífilis son más altas en cada una de las variables estudiadas (edad, educación, estado civil, CPN, paridad, bajo peso al nacer previo, y muerte perinatal previa).

El análisis de regresión múltiple muestra que la ausencia de educación, bajo número de control prenatal, la mayor paridad, el ser conviviente o soltera, el tener antecedente de niño nacido con bajo peso y el vivir en la selva son factores asociados a la positividad en la prueba de sífilis. El vivir en la sierra resulta ser protector. La mayor significancia se observa con el antecedente de muerte perinatal (ORa=2,13 IC 95\% 1,88-2,41) (Tabla 4).

\section{DISCUSIÓN}

La cobertura de tamizaje para sífilis materna se ha incrementado de manera significativa y, en promedio, alcanza para el país $80,7 \%$. Esto es debido a mayor y mejor acceso a servicios de salud y a un mayor número de controles prenatales. La mayor cobertura es en la selva $(87,7 \%)$ y esto es importante pues en dicha región se observa la mayor prevalencia de sífilis materna. Un diagnóstico oportuno permitirá un tratamiento adecuado.

Para el periodo $2000-2010$ se registra una prevalencia promedio de $0,70 \%$ de sífilis gestacional un valor menor al $1,71 \%$ observado en la década de los noventa ${ }^{(10)}$.

En la región de América Latina y el Caribe, la sífilis es la infección de transmisión sexual (ITS) de menor prevalencia comparadas con las ITS clásicas ${ }^{(14)}$. Según los datos suministrados a la OPS por los programas nacionales de ITS/VIH/SIDA3 durante el año $2002{ }^{(15)}$, se observa que la prevalencia estimada de sífilis en gestantes en la región es de 3,1\%. El Perú tiene una de las prevalencias más bajas $(1,0 \%)$ junto con Panamá. En el otro espectro, los valores más altos se encuentran en Paraguay (6,1\%), y prevalencias algo menores en El Salvador, Bolivia y Brasil. En el caso de la sífilis congénita el Perú se encuentra en tercer lugar de diez países de la región. La incidencia más alta se observa en Honduras con 12 casos por 1000 nacidos vivos.

En el presente estudio, al revisar la base de datos del SIP entre el 2000 y 2010 , se encuentra que las prevalencias más altas de sífilis materna se observan en la selva alta $(1,13 \%)$ y en la selva baja $(1,30 \%)$. Esta asociación se mantiene en el análisis multivariable luego de controlar por variables como edad, educación, control prenatal, paridad, estado civil, antecedente de niños con bajo peso al nacer, muerte perinatal previa y edad gestacional a la presencia de sífilis. Esta situación es preocupante por los hábitos de conducta sexual de los pobladores de la selva del Perú. Más aun, estudios en comunidades nativas amazónicas realizadas en 2004 muestran prevalencias de sífilis del orden de $3,2 \%$ (3,7\% para varones y $2,7 \%$ para mujeres) ${ }^{(12)}$. Igualmente, otros estudios tanto a nivel urbano como rural muestran la mayor prevalencia de sífilis en la Amazonía peruana (16,17). Esto implica la necesidad de una urgente atención de salud pública en las poblaciones de la Amazonía. La sierra en relación con la costa, presenta más bien un efecto protector donde el OR para positividad de serología positiva de sífilis es menor que en la costa.

La menor prevalencia de sífilis materna en la sierra es concordante con una observación anterior de menor prevalencia de sífilis en varones y mujeres de la 
sierra del Perú ${ }^{(16)}$. No se sabe, a la fecha, si la menor prevalencia está asociado a diferencias conductuales de actividad sexual.

En el Perú (10), la prevalencia de sífilis materna ha variado de $1,7 \%$ en 1996 , a $1,0 \% ; 1,5 \% ; 1,6 \% ; 1,4 \%$ en los años 1997, 1998, 1999 y 2000 . El porcentaje de tamizaje de prueba Regina Plasmática Rápida (RPR) que identifica la presencia de anticuerpos no específicos contra Treponema pallidum ha ido en aumento de 35,9\% en 1997 a 75,7\% en 2005. Para el 2002, de acuerdo al MINSA, la tasa de sífilis materna en el Perú se encuentra en 0,56\% (IC 95\%: 0,43-0,73) con diferencias entre regiones geográficas, $0,19 \%(0,09-0,35)$ en la costa, $0,94(0,65-1,32)$ en la sierra y $0,95(0,52-1,60)$ en la selva. Para el mismo año este estudio reporta prevalencias de $0,6 \%$ para costa, $0,8 \%$ para sierra y $1,9 \%$ para selva.

El presente estudio abarca el $31-37 \%$ de hospitales públicos del país; sin embargo, ello no afectaría los resultados dado que se están incluyendo en el análisis ciudades de la costa, sierra y selva (selva alta y baja) y del norte, centro y sur del país. En la población general la prevalencia de sífilis para 2002 es de $0,5 \%$ en varones, y $0,4 \%$ en mujeres ${ }^{(16)}$; valores muy cercanos a los observados para sífilis materna en el mismo año $(0,56)$. Es interesante el resultado que el antecedente de muerte perinatal previa presente el OR más alto de todas las variables estudiadas, lo que indicaría la posibilidad de una cronicidad de la sífilis.

Es importante el hallazgo de una reducción de las prevalencias de sífilis materna observadas durante el último decenio, que contrasta a lo observado en otros países como es el caso de México, donde se observa una reemergencia de la sífilis materna y congénita, particularmente en las zonas del Norte mexicano ${ }^{(18)}$.

La OMS ha propuesto una iniciativa para erradicar la sífilis materna y la sífilis congénita; sin embargo, aún dista mucho de lograrse. Se han hecho muchos esfuerzos a través de los países miembros en mejorar el tamizaje de sífilis materna y neonatal. Son claros los efectos en que una mayor cobertura de tamizaje se asocia también a una disminución en las prevalencias de sífilis materna. Aún existe mucho trabajo por hacer para disminuir las prevalencias de sífilis congénita, en tanto su eliminación se puede lograr a través de "la implementación de una serie de medidas probadas pero requiere de soporte técnico, fondos, y un compromiso entre las fuerzas políticas, funcionarios de salud, y el público, para prevenir y tratar todos los casos de sífilis congénita y ayudar a los países a lograr su meta de desarrollo del milenio ${ }^{(19)}$.
El tamizaje de sífilis antenatal acompañado al de otras enfermedades transmisibles como HIV y hepatitis B ha resultado en dramática reducción de infección vertical al neonato en países desarrollados, acompañado a un bajo porcentaje de rechazo a las pruebas ${ }^{(20)}$. En Alberta, Canadá, se demostró en un estudio sobre sífilis materna, que los infantes con sífilis congénitas nacen de madres con sífilis infecciosa que tienen limitado control prenatal (21). Es importante, por ello, que aquellas mujeres en alto riesgo para sífilis gestacional acudan a los centros médicos para la atención prenatal.

En el Perú, las normas del Ministerio de Salud indican que toda gestante que acuda a un establecimiento de salud para su atención prenatal debe recibir consejería pretest y proceder a tomar la muestra de sangre para el tamizaje con ELISA o prueba rápida para descartar infección por VIH; así mismo, la prueba de RPR para descartar la infección por Treponema pallidum, de preferencia en la primera atención prenatal. Según la norma del MINSA 2008, se debe realizar el cribado para sífilis durante la atención del parto, el puerperio o el aborto.

En nuestro país están teniendo resultados los planes del Ministerio de Salud y cada vez se observa un incremento en el número de visitas prenatales a los servicios médicos. Sin embargo, aún hay brechas que cerrar. Las poblaciones en las zonas de la selva y sierra aún tienen menos acceso a los servicios de salud y acceden en menor proporción al control prenatal desde el primer trimestre de gestación ${ }^{(22)}$. En nuestro estudio se muestra que uno de los factores que contribuye con una mayor prevalencia de sífilis materna es el bajo nivel educativo de la madre. Igualmente, el hecho de habitar en la selva también aporta de manera importante en aumentar la prevalencia de sífilis gestacional. Las conductas de riesgo contribuyen de manera importante en la prevalencia de la enfermedad (23).

Una limitación de la base SIP es que no indica sobre la existencia de tratamiento en casos de serología positiva para lúes. En la base SIP no figuran datos de tratamiento profiláctico para sífilis materna y de sus parejas. Ello indica la necesidad de mejorar la ficha del SIP incorporando lo relativo al tratamiento. Igualmente, es necesario mejorar la evaluación del recién nacido en términos de tamizaje de sífilis. EL SIP, a pesar de estar registrado en diferentes centros de salud en el Perú, tiene un uso o aplicación para monitoreo y vigilancia escaso. Hay experiencias favorables con el uso del SIP en la sífilis gestacional, como la observada en Uruguay ${ }^{(6)}$.

Entre las limitaciones en el cálculo de las prevalencias de sífilis se encuentran que las pruebas diagnósticas pueden diferir entre países. También queda pendiente el 
caso de la subnotificación. El Ministerio de Salud del Perú en $2007^{(10)}$ estableció diferentes planes que apuntan a reducir o erradicar la sífilis materna y congénita en el país. Esto aún no ha ocurrido en su totalidad, aunque los esfuerzos han sido positivos pues se ha mejorado en el último decenio la cobertura. EI SIP puede ser una herramienta importante para el monitoreo ${ }^{(23)}$.

En conclusión, los resultados del presente estudio muestran que las prevalencias de sífilis materna han disminuido durante la década 2000-2010; sin embargo, la población de la selva a lo largo de los 10 años muestra mayor prevalencia, y los de la sierra tienen la menor prevalencia. El tamizaje para sífilis es más alto en la selva que en la costa y sierra. No hay asociación entre porcentaje de tamizaje y prevalencia de seropositividad para sífilis en ninguna de las regiones geográficas. Los factores de riesgo asociados a la presencia de sífilis son la ausencia de educación, bajo número de controles prenatales, la multiparidad y, en mayor proporción, el antecedente de una muerte perinatal previa.

Agradecimientos: a la Oficina Panamericana de la Salud (OPS), Lima y al Centro Latinoamericano de Perinatología y Desarrollo Humano (CLAP, OPS), al Dr. Adrián Díaz por el apoyo brindado, a los hospitales del Ministerio de Salud que facilitaron el acceso a las bases de datos del SIP

Contribuciones de autoría: GFG ha participado en la concepción, diseño y redacción del artículo. VT ha participado en el análisis e interpretación de datos y revisión del artículo. SS ha participado en el análisis de la información y revisión crítica del artículo.

Fuentes de financiamiento: Oficina Panamericana de la Salud (OPS), Lima.

Conflictos de interés: los autores declaran no tener conflictos de interés.

\section{REFERENCIAS BIBLIOGRÁFICAS}

1. Schmid G, Rowley J, Samuelson J, Tun Y, Guraiib M, Mathers C, et al. Global incidence and prevalence of four curable sexually transmitted infections (STIs): New estimates from WHO. In: Proceedings of the 2nd Global HIV/ AIDS Surveillance Meeting (ISSTDR '09), London, UK, June-July 2009.

2. Schmid GP, Stoner BP, Hawkes S, Broulet N. The need and plan for global elimination of congenital syphilis. Sex Transm Dis. 2007 Jul;34(7 Suppl):S5-10.

3. Berman SM. Maternal syphilis: pathophysiology and treatment. Bull World Health Organ. 2004 Jun;82(6):433-8.

4. Terris-Prestholt F, Watson-Jones D, Mugeye K, Kumaranayake L, Ndeki L, Weiss H, et al. Is antenatal syphilis screening still cost-effective in sub-Saharan Africa. Sex Transm Infect. 2003 Oct;79(5):375-81.

5. Kamb ML, Newman LM, Riley PL, Mark J, Hawkes SJ, Malik T, et al. A road map for the global elimination of congenital syphilis. Obstet Gynecol Int. 2010;2010. doi: $10.1155 / 2010 / 312798$.

6. López Gómez A, Benia W, Alemán A, Vásquez J. Una década de sífilis gestacional y Congénita en Uruguay: 2000-2009. Montevideo: OPS CLAP/ SMR; 2011.
7. Salmavides-Cuba F, Banda-Flores CL. Sífilis en la gestación. Rev Per Ginec Obst. 2010;56:202-8.

8. NIMH Collaborative HIV/STD Prevention Trial Group. Sexually transmitted disease and HIV prevalence and risk factors in concentrated and generalized HIV epidemic settings. AIDS 2007 Apr;21 Suppl 2:S81-90.

9. García PJ, Chavez S, Feringa B, Chiappe M, Li W, Jansen KU, et al. Reproductive tract infections in rural women from the highlands, jungle, and coastal regions of Peru. Bull World Health Organ. 2004 Jul;82(7):483-92.

10. Perú, Ministerio de Salud. Plan nacional de prevención y control de la transmisión madre - niño de VIH y sífilis: 2007-2011 / Ministerio de Salud. Dirección General de Salud de las Personas. Estrategia Sanitaria Nacional de Prevención y Control de Infecciones de Transmisión Sexual y VIH/SIDA -- Lima: Ministerio de Salud; 2007.

11. Gonzales GF. La adolescencia en el Perú. Lima: UPCH 1994:1-324.

12. Bartlett EC, Zavaleta C, Fernández C, Razuri H, Vilcarromero S, Vermund $\mathrm{SH}$, et al. Expansion of HIV and syphilis into the Peruvian Amazon: a survey of four communities of an indigenous Amazonian ethnic group. Int J Infect Dis. 2008 Nov;12(6):e89-94. doi: 10.1016/j.ijid.2008.03.036.
13. Clark JL, Konda KA, Munayco CV, Pún M, Lescano AG, Leon SR, et al. Prevalence of HIV, herpes simplex virus-2, and syphilis in male sex partners of pregnant women in Peru. BMC Public Health. 2008 Feb 19;8:65. doi: 10.1186/1471-2458-8-65.

14. Gerbase AC, Toscano C, Titan S, Cuchí P, González-Salvatierra R, Zacarías F. Sexually transmitted diseases in Latin America and the Caribbean. Rev Panam Salud Publica. 1999;6(5):362-70.

15. Valderrama J, Zacarías F, Mazin R. Maternal syphilis and congenital syphilis in Latin America: big problem, simple solution. Rev Panam Salud Publica. 2004 Sep;16(3):211-7. [Article in Spanish]

16. Cárcamo CP, Campos PE, García PJ, Hughes JP, Garnett GP, Holmes KK, et al. Prevalences of sexually transmitted infections in young adults and female sex workers in Peru: a national population-based survey. Lancet Infect Dis. 2012 Oct;12(10):765-73. doi: 10.1016/S1473-3099(12)70144-5.

17. Zavaleta C, Fernández C, Konda K, Valderrama Y, Vermund SH, Gotuzzo E. High prevalence of HIV and syphilis in a remote native community of the Peruvian Amazon. Am J Trop Med Hyg. 2007 Apr;76(4):703-5.

18. Reyna-Figueroa J, Esparza-Aguilar M, Hernández-Hernández Ldel $\mathrm{C}$, Fernández-Canton S, Richardson- 
Lopez Collada VL. Congenital syphilis, a reemergent disease in Mexico: its epidemiology during the last 2 decades. Sex Transm Dis. 2011 Sep;38(9):798-801. doi: 10.1097/ OLQ.0b013e31821898ca.

19. Hossain M, Broutet N, Hawkes S. The elimination of congenital syphilis: a comparison of the proposed World Health Organization action plan for the elimination of congenital syphilis with existing national maternal and congenital syphilis policies. Sex Transm Dis. 2007 Jul;34(7 Suppl):S22-30.

20. Op de Coul EL, Hahné S, van Weert YW, Oomen P, Smit C, van der Ploeg KP, et al. Antenatal screening for HIV, hepatitis B and syphilis in the Netherlands is effective. BMC Infect Dis. 2011 Jun 30;11:185. doi: 10.1186/1471-2334-11-185.

21. Caddy SC, Lee BE, Sutherland K, Robinson JL, Plitt SS, Read R, et al. Pregnancy and neonatal outcomes of women with reactive syphilis serology in Alberta, 2002 to 2006. J Obstet Gynaecol Can. 2011 May;33(5):453-9.

22. Instituto Nacional de Estadística e Informática. Perú: Indicadores de resultados de los Programas Estratégicos, 2010. Encuesta Demográfica y de Salud Familiar - ENDES Continua. Lima: INEI; 2011.

23. Alarcón JO, Johnson KM, Courtois B, Rodríguez C, Sanchez J, Watts DM, et al. Determinants and prevalence of HIV infection in pregnant Peruvian women. AIDS 2003 Mar 7;17(4):613-8.

24. Organización Panamericana de la Salud. Iniciativa Regional para la eliminación de la transmisión materno infantil del VIH y de la Sífilis Congénita en América Latina y el Caribe: documento conceptual. Montevideo: CLAP/ SMR; 2009.

Correspondencia: Gustavo F. Gonzales Dirección: Universidad Peruana Cayetano Heredia. Av. Honorio Delgado 430. Lima 31, Perú.

Correoelectrónico:gustavo.gonzales@upch.pe
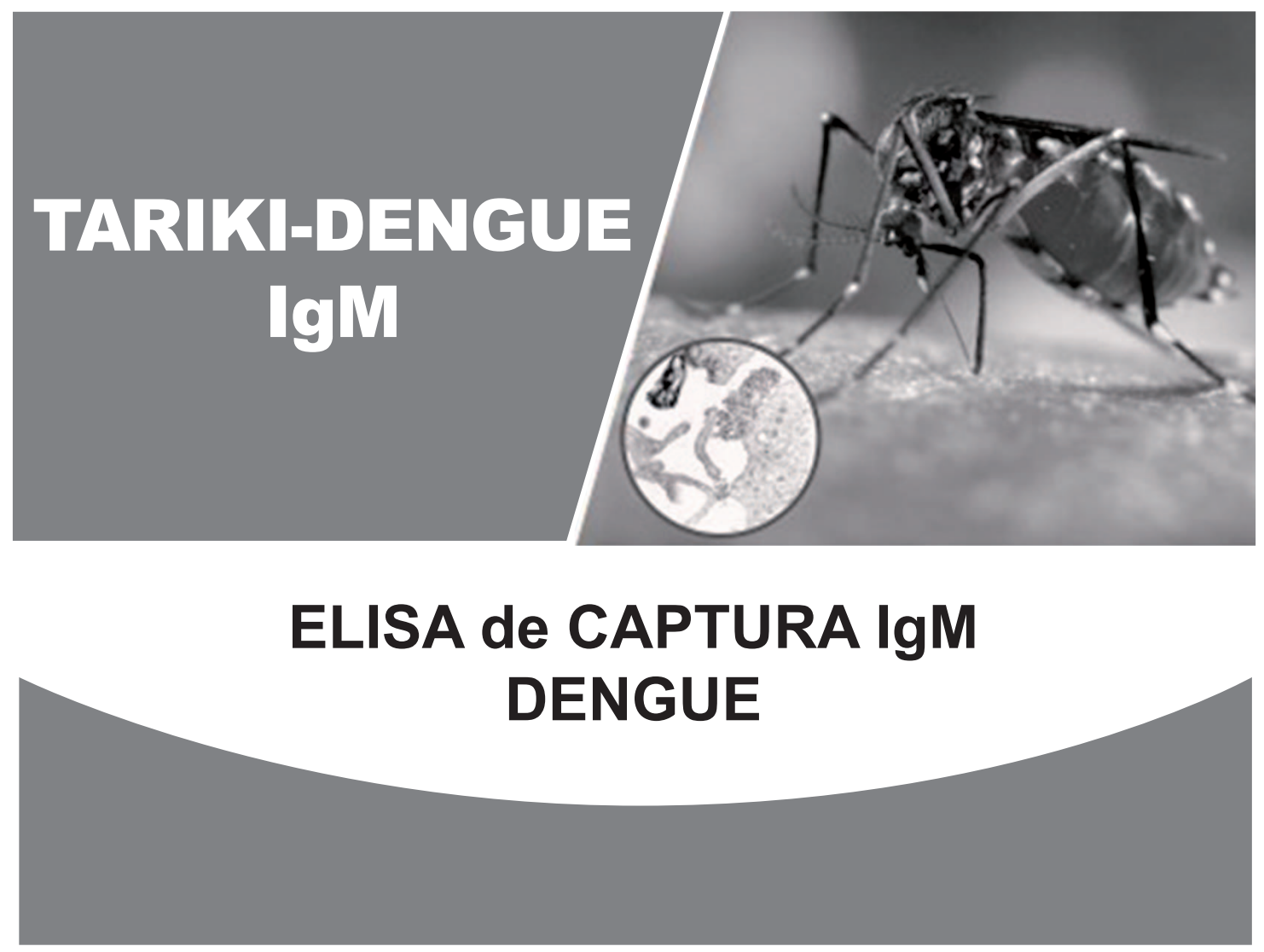

TARIKI: disponible en el Perú

Kit para la determinación de anticuerpos IgM anti Dengue, desarrollado y producido por el Instituto Nacional de Salud, Perú INS/MINSA 